\title{
In-situ Heating in SEM and FIB/SEM Systems
}

\author{
Petr Wandrol ${ }^{1}$, Libor Novák ${ }^{1}$, Min Wu ${ }^{1}$, Ernst Jan Vesseur ${ }^{2}$ and Tomáš Vystavěl ${ }^{1}$ \\ 1. Thermo Fisher Scientific, Materials \& Structural Analysis, Brno, Czech Republic. \\ 2. Thermo Fisher Scientific, Materials \& Structural Analysis, Eindhoven, the Netherlands.
}

Rapid growth of interest in the in-situ transmission electron microscopy (in-situ TEM) has been seen within the scientific community over the last decade. In contrast, in-situ or environmental scanning electron microscopy (in-situ SEM, ESEM) has received less interest due to its limited performance for use cases requested in the materials research. Significant innovations in the area of sample heating presented recently [1] opened up new application possibilities and improved ease of use of the in-situ SEM. This is expected to boost interest by allowing researchers to better study processes at surfaces and at the length scales that only SEM can address. This offers unique opportunity to scientists who can choose a heating module with respect to the sample type and experiment design and hence to significantly improve quality of acquired data or achieve unique results that were unreachable in the past. This contribution presents insitu experiments in the SEM and FIB/SEM using several types of heating stages under gaseous or high vacuum environment in the specimen chamber. Processes taking place at high temperatures are characterized by the means of secondary (SE), backscattered (BSE) and transmitted electrons (STEM). Energy dispersive spectroscopy (EDS) and electrons backscatter diffraction (EBSD) is employed to monitor heat induced changes in elemental composition and crystallography.

Exploring dynamic response of a sample to the temperature under gaseous environment in the ESEM chamber is a time-proven method. The main advantage of the ESEM is a homogeneous distribution of gas in the specimen chamber and hence around the sample allowing studying reactions such as oxidation or reduction using water vapor or forming gas $\left(5 \%\right.$ of $\mathrm{H}_{2}$ in $\left.\mathrm{N}_{2}\right)$. Example of a typical experiment showing recrystallization of platinum at a temperature of $900^{\circ} \mathrm{C}$ and $400 \mathrm{~Pa}$ of water vapor in the chamber of Thermo Scientific Quattro S field emission SEM is shown in Figure 1. Another approach is to inject a reactive gas into the proximity of the area of interest while the chamber stays in high vacuum [2]. This approach is possible with the newly developed High Vacuum Heating Stage which is compatible with a chamber pressure down to $10^{-4} \mathrm{~Pa}$. The module ensures cleanliness of the experiment and benefits from using standard detectors including in-lens. Moreover, its design enables one to tilt up to $70^{\circ}$ for in-situ phase transformation and recrystallization studies by EBSD.

Further extension of the application space towards rapid changes of temperature, full detector compatibility and ultra-high resolution imaging requires change of the heater technology. Novel microheatingplate device ( $\mu$ Heater) based on microelectromechanical systems (MEMS) technology provides extraordinary heating rates in the order of $10^{4}$ kelvin per second with maximal temperature of $1200^{\circ} \mathrm{C}$ [3]. Typical samples that can be accommodated at the $\mu$ Heater active area are either particles with the size ranging from nanometers to tens of microns or FIB prepared blocks transferred from a bulk sample to the heated area in-situ in the FIB/SEM system. Thanks to the design of the $\mu$ Heater device, STEM imaging and EDS analysis of heated samples with minimized influence of thermal radiation become possible on top of SE, BSE and EBSD collection that was presented earlier [4]. Benefits of using the STEM to image in-situ heating experiments is demonstrated by high resolution observation of coalescence of palladium nanoparticles on carbon while heating from $500^{\circ} \mathrm{C}$ to $1100^{\circ} \mathrm{C}$ in Figure 2 . 
In conclusion, the application potential of in-situ SEM has been significantly broadened by the development of new types of heating stages. Choosing the appropriate heating module with respect to the sample type and design of the experiment significantly improves the quality of experimental data. Capabilities in signal detection and ultra-high resolution imaging have been greatly increased. Last but not least, the integration of all heating modules into the SEM User Interface sharing identical control elements and philosophy has very much improved the ease of use of in-situ SEM [5].

\section{References:}

[1] L. Novák et al, Microsc. Microanal. 23 (S1) (2017), p. 928.

[2] M. Kolíbal et al, Nano Lett. 16(8) (2016), p. 4880.

[3] L. Mele et al, Microscopy Research and Technique 79 (2016), p. 239.

[4] L. Novák et al, Microsc. Microanal. 22 (S3) (2016), p. 184.

[5] The authors acknowledge funding from Tech. Agency of the Czech Republic, TE01020118.
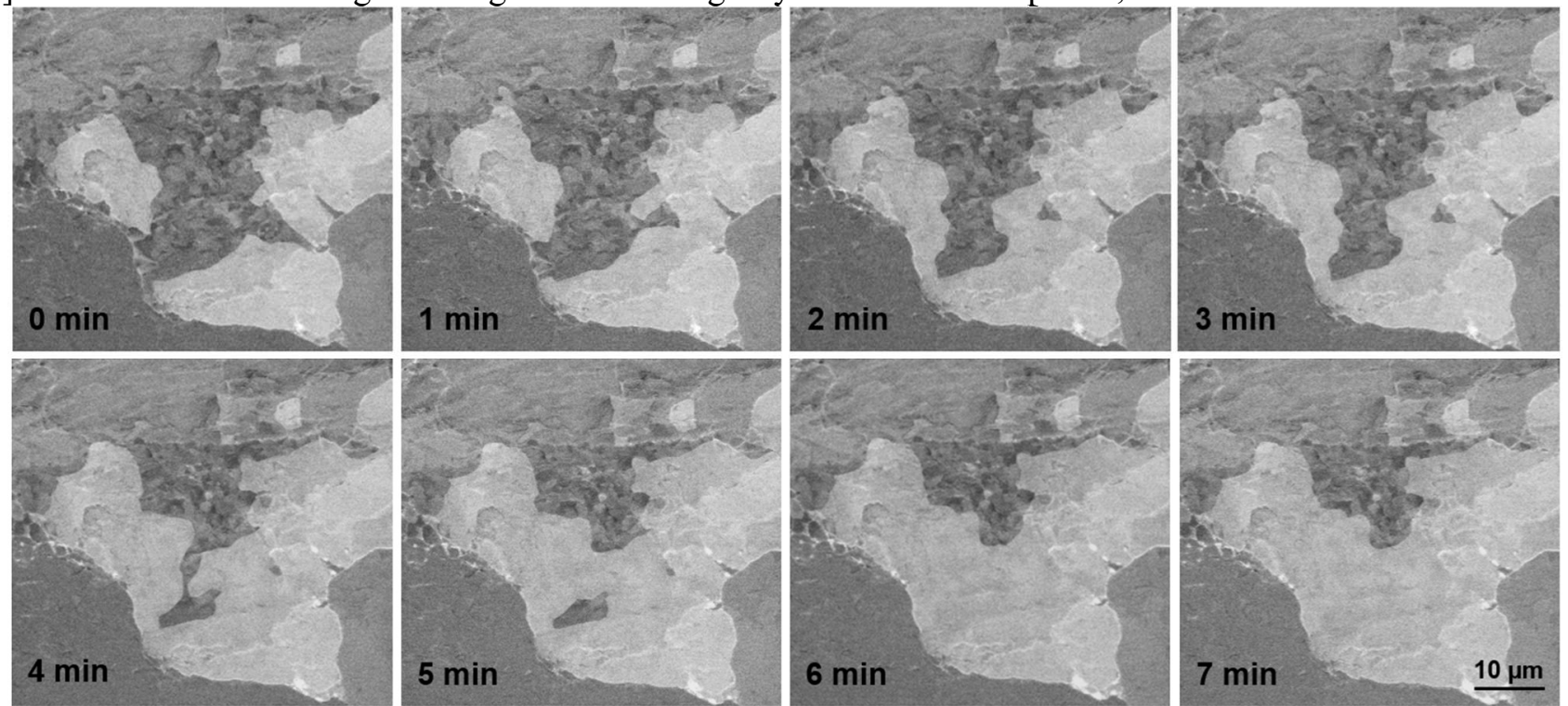

Figure 1. Recrystallization of platinum at the temperature of $900^{\circ} \mathrm{C}$ and $400 \mathrm{~Pa}$ of water vapor.
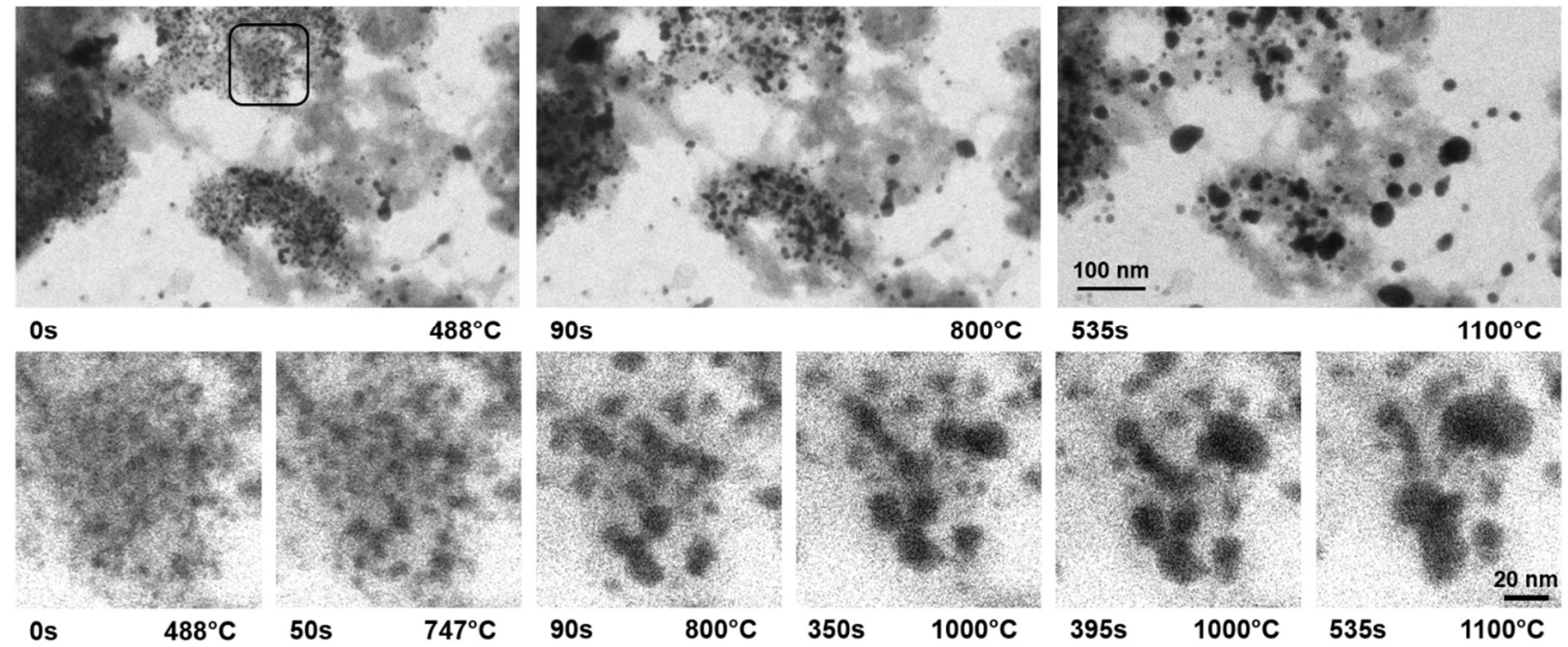

Figure 2. Bright field STEM images of a coalescence of Pd nanoparticles on carbon while heating from 500 to $1100^{\circ} \mathrm{C}$ by the $\mu$ Heater. Magnified area is indicated by the rectangle in the first image. 